\title{
Assessment of the Efficacy of Interventions for the Treatment of Sleep Respiratory Disorder in Chronic Heart Failure Patients: A Systematic Review
}

\author{
Kronik Kalp Yetersizliği Hastalarında Uykuda Solunum Bozukluğunun Tedavisi İçin \\ Kullanılan Girișimlerin Etkinliğinin Değerlendirilmesi: Sistematik Derleme
}

(D) Hilal Uysal11, (D) Hanım Büşra Oruçoğlu²

${ }^{1}$ istanbul University-Cerrahpaşa Florence Nightingale Faculty of Nursing, Department of Internal Medicine Nursing, istanbul, Turkey

2istanbul University-Cerrahpașa Florence Nightingale Faculty of Nursing, i̇stanbul, Turkey

\begin{abstract}
One of the most important problems of heart failure (HF) patients is sleep disturbance. In HF patients with obstructive and central sleep apnea, hypoxia, hypercapnia and overexcitation of the sympathetic system are observed. As a result, negative intrathoracic pressure and left ventricular afterload increase. Treatment of sleep respiratory disorders in chronic HF patients is important for the prognosis of the disease. Therefore, in this systematic review, we aimed to evaluate the efficacy of interventions used for the treatment of sleep respiratory disorder in chronic HF patients.

Cochrane Library, Scopus, Springer Link, Science Direct, Clinical Key, PubMed, Turkey Citation Index and EBSCO databases were searched for studies between June 2017-August 2018. When all studies were examined, out of 2.691 .006 studies published between 2007-2017, 16 randomized controlled trials that met inclusion criteria were included in the study. In these studies, treatment interventions for treatment groups with sleep respiratory disorder included continuous positive airway pressure (CPAP), bi-level positive airway pressure, adaptive servo-ventilation (ASV), atrial overdrive pacing, home oxygen therapy, slow breathing exercise device and structured physical exercise. When the study results are examined, CPAP treatment improved daytime sleepiness and left ventricular ejection fraction (LVEF) but did not provide significant improvement on quality of life, and that ASV treatment reduced apneahypopnea index, provided improvement in LVEF and cardiac function, and reduced ventricular ejection fraction. However, further research is needed to fully demonstrate the efficacy of interventions for the treatment of sleep respiratory disorder in chronic HF patients.
\end{abstract}

Keywords: Sleep apnea, obstructive sleep apnea, central sleep apnea, heart failure öz

Kalp yetersizliği hastalarının en önemli sorunlarından birisi uyku bozukluğudur. Obstrüktif ve santral uyku apnesi șikayeti olan kalp yetersizliği hastalarında hipoksi, hiperkapni ve sempatik sistemin aşırı uyarılması söz konusudur. Bunun sonucunda negatif intratorasik basınç ve sol ventrikül ard yükü artar. Kronik kalp yetersizliği hastalarında uyku bozukluğunun tedavisi hastalığın prognozu için önemlidir. Bu nedenle bu sistematik derleme çalışmasında, kronik kalp yetersizliği hastalarında uykuda solunum bozukluğunun tedavisi için kullanılan girişimlerin etkinliğinin değerlendirilmesi amaçlandı. Cochrane Library, Scopus, Springer Link, Science Direct, Clinical Key, PubMed, Türkiye Atıf Dizini ve EBSCO'da yer alan çalıșmalar Haziran 2017-Ağustos 2018 tarihleri arasında incelendi. Tüm çalıșmalar incelendiğinde, toplam taranan 2.691 .006 veriden 2007-2017 yılları arasında yayımlanmış, kronik kalp yetersizliği ve uykuda solunum bozukluğu olan (sol ventrikül ejeksiyon fraksiyon $\leq \% 45$, apne-hipopne indeksi $>10 /$ saat), en az 3 ay takip edilmiș hastaların dahil edildiği 16 randomize kontrollü çalıșma araștırmaya dahil edildi.

Çalıșmalarda uykuda solunum bozukluğu olan girișim grubu için tedavi girișimleri olarak devamlı pozitif havayolu basıncı, iki seviyeli pozitif havayolu basıncı, adaptif servo-ventilasyon, overdrive pacemaker, evde oksijen tedavisi, yavaş solunum egzersizi cihazı ve yapılandırılmış fiziksel egzersiz girişimi uygulandığı tespit edildi. Çalıșma sonuçları incelendiğinde, devamlı pozitif havayolu basınc tedavisinin gündüz uykuluk durumunu ve sol ventrikül ejeksiyon fraksiyonu iyileștirdiği, ancak yaşam kalitesi üzerine anlamlı iyileșme sağlamadığı, adaptif servo-ventilasyon tedavisinin apne-hipopne indeksini azalttığı, sol ventrikül ejeksiyon fraksiyonda ve kardiyak fonksiyonlarda iyileșme sağladığı, ventrikül atım sayısını azalttığı tespit edilmiștir. Ancak kronik kalp yetersizliği hastalarında uykuda solunum bozukluğunun tedavisi için uygulanan girișimlerin etkisinin tam olarak ortaya konulabilmesi için daha fazla araştırmaya intiyaç olduğu ortaya çıkmaktadır.

Anahtar Kelimeler: Uyku apnesi, obstrüktif uyku apnesi, santral uyku apnesi, kalp yetersizliği
Address for Correspondence/Yazıșma Adresi: Hilal Uysal MD, İstanbul University-Cerrahpaşa Florence Nightingale Faculty of Nursing, Department of Internal Medicine Nursing, İstanbul, Turkey

Phone: +90 2124400000 E-mail: hilaluysal@gmail.com ORCID ID: orcid.org/0000-0003-3211-7011

Cite this article as/Atıf: Uysal H, Oruçoğlu HB. Assessment of the Efficacy of Interventions for the Treatment of Sleep Respiratory Disorder in Chronic Heart Failure Patients: A Systematic Review. İstanbul Med J 2019; 20(3): 176-87.

(c) Copyright 2019 by the Istanbul Training and Research Hospital/Istanbul Medical Journal published by Galenos Publishing House.

(C) Telif Hakkı 2019 Istanbul Ĕgitim ve Araștırma Hastanesi/Istanbul Tıp Dergisi, Galenos Yayınevi tarafından basılmıștır.
Received/Geliș Tarihi: 28.08.2018 Accepted/Kabul Tarihi: 12.11.2018 


\section{Introduction}

Heart failure (HF) is defined as a structural or functional cardiac disorder that causes the heart not to provide enough oxygen to meet the metabolic needs of the tissues despite the normal filling pressures. Approximately $1-2 \%$ of the adult population in developed countries has HF. The prevalence of HF is reported to be $10 \%$ or more in individuals aged 70 years and older (1).

Sleep disorder is one of the most important problems of HF patients. Hypoxia, hypercapnia and over-excitation of the sympathetic system are present in HF patients with obstructive and central sleep apnea (CSA). As a result, negative intrathoracic pressure and left ventricular afterload increase. Determination of the degree of sleep disturbance by polysomnography (PSG) and administration of oxygen therapy, continuous positive airway pressure (CPAP), bi-level (BI) PAP for the treatment of obstructive sleep apnea (OSA) and adaptive servoventilation methods (ASV) for the treatment of nocturnal hypoxemia during the night are recommended in patients with HF (1).

The degree of SRD is determined by PSG and OSA and/or CSA classification is done. The hourly apnea-hypopnea index (AHI) between 5-15 is classified as mild OSA, between 15-30 as moderate OSA, and $>30$ as severe OSA. In the literature, it has been shown that patients with OSA with an AHI value of $>20$ have increased risk of morbidity and mortality if not treated (2). The diagnostic criteria of primary CSA syndrome are as follows: a) At least one of the following from frequent arousals and awakenings during sleep, excessive daytime sleepiness and awakening short of breath; b) PSG shows $\geq 5$ central apneas per hour of sleep, representing $>50 \%$ of total respiratory events in the AHI; and c) the disorder is not better explained by another current sleep disorder, a medical or neurologic disorder, medication use, or a substance use disorder. The diagnostic criteria of Cheyne-Stokes respiration (CSR) include $\geq 5$ central apneas-hypopneas during sleep and monitoring crescendo-decrescendo cycle over a period of at least 10 minutes (3).

The gold standard treatment of OSA is PAP. The aim of the treatment of SRD is to ensure that the upper airway remains open during sleep and to regulate quality of breathing and sleep. However, the patient benefits from this treatment during the time she/he uses the device. For this reason, it is recommended that the patient should use the device over $70 \%$ and more than 4 hours during the night $(2,3)$.

As reviewed in the literature, the treatment of sleep disturbance in chronic (C) HF patients is important for the prognosis of the disease. Therefore, the aim of this systematic review was to evaluate the efficacy of interventions for the treatment of SRD in CHF patients.

\section{Methods}

This systematic review was planned and carried out as a descriptive study to evaluate the efficacy of interventions used for the treatment of SRD in CHF patients. The following steps were used in systematic review. The framework of the study was based on (population, intervention, comparator, outcome and study design) (4). These steps are described in detail in Table 1.

Inclusion criteria were RCTs published between 2007-2017, including patients with CHF and RSD (OSA, CSA) (left ventricular ejection fraction (LVEF) $\leq 45 \%$, AHI > 10/hour) followed for at least 3 months (Table 1).
All of the RCTs included in the study were evaluated by two researchers using an appropriate quality assessment checklist (5). The results of the evaluation were discussed and the publications prepared according to the RCT criteria were included in the study.

In data extraxtion analysis, one of the researchers first examined the researches included and collected data on the findings and characteristics of the research. The second researcher then checked the accuracy of this data. Data extraction steps were carried out by the authors. The data extraction steps and the findings are explained in detail in Table 2.

In this study, the answer to the question "Which interventions are effective in the treatment of SRD in patients with CHF?" was investigated.

No funding was received during the research. Researchers used their own means throughout the study. The researchers took part in each phase of the study according to the timetable. The timetable of the study was carried out as follows:

1. Preparation of the research protocol: 07/06/2017

2. Scanning period: June-September 2017

\section{Analysis: October 2017-March 2018}

\section{Article writing: April-August 2018}

In the study, keywords were selected in accordance with the research topic (Figure 1) and electronic scanning was performed. In the selection of keywords, attention was paid to the scanning of the full text of RCTs, in which the effectiveness of interventions used in the treatment of $\mathrm{CHF}$ patients diagnosed with SRD.

Cochrane Library, Scopus, Springer Link, Science Direct, Clinical Key, PubMed, EBSCO and Turkey Citation Index electronic databases were used for scanning. Medline, EMBASE, Ovid and CINAHL databases were not used because they could not be accessed. When all the studies recorded according to inclusion criteria were examined, 16 publications from total scanned 2.691.006 data were included in our study (Figure 1) (6).

\begin{tabular}{l} 
Table 1. Inclusion and exclusion criteria \\
Inclusion criteria \\
- Published between $2007-2017$ \\
\hline - Followed for at least 3 months \\
\hline - Randomized controlled trials \\
\hline - Diagnosed with chronic heart failure \\
- LVEF $\leq 45 \%$ \\
\hline - Diagnosed with CSA and/or OSA \\
\hline - AHI >10/hour \\
\hline - Diagnosis of sleep respiratory disorder by polysomnography \\
\hline - Research in English and Turkish \\
\hline - Full texts \\
\hline - Studies on specified keywords \\
\hline Exclusion criteria \\
\hline - Studies not published in Turkish and English \\
\hline - Unpublished thesis studies \\
\hline - Reviews \\
\hline - Abstracts \\
\hline - Conference summaries, protocols, case reports, in vitro studies \\
\hline - Ongoing study findings \\
\hline LVEF: left ventricular ejection fraction, CSA: central sleep apnea, OSA: obstructive sleep \\
apnea, AHI: apnea-hypopnea index
\end{tabular}




\begin{tabular}{|c|c|}
\hline Number of articles cran & 10001030 \\
\hline $\begin{array}{l}\text { Keywords } \\
\text { English: Heart failure, chronic heart } \\
\text { failure, polisomnography, sleep } \\
\text { disorders, sleep apnea, sleep quality, } \\
\text { obstructive sleep apnea, central aleep } \\
\text { apnea. } \\
\text { Turkish: Kalp Yetersizliği, kronik } \\
\text { kalp yetersizliği, polisomnografi, } \\
\text { uyku uyku bozukluğu, uyku apnesi, } \\
\text { uyku kalitesi, obstruktif uyku apnesi, } \\
\text { santral uyku apnesi. }\end{array}$ & $\begin{array}{l}\text { Number of articles scanned by words in } \\
\text { databases } \\
\text { CLINICAL KEY: } 95.666 \\
\text { COCHRANE LIBRARY: } 573 \\
\text { EBSCO: } 1098 \\
\text { SCIENCE DIRECT: } 1.071 .673 \\
\text { SCOPUS: } 530.056 \\
\text { SPRINGER LINK: } 705.691 \\
\text { PUBMED: } 286.854 \\
\text { TURKIYE CITATION INDEX: } 803\end{array}$ \\
\hline $\begin{array}{l}\text { Therapy initiatives for sleep- } \\
\text { disordered breathing included in } \\
\text { the study: CPAP, BIPAP, Adaptive } \\
\text { Servo Ventilation (ASV), atrial } \\
\text { overdrive pacemaker, oxygen therapy } \\
\text { at home (HOT), slow breathing } \\
\text { exercise device (RESPERATE) and } \\
\text { physical exercise therapy: } 16\end{array}$ & $\begin{array}{l}\text { Exclusion criteria } \\
\text { Unpublished and not followed for at least } \\
3 \text { months between } 2007 \text { and } 2017: 13.454 \\
\text { Other than randomized controlled study: } \\
18.802 .898 \\
\text { Patients without a diagnosis of chronic } \\
\text { heart failure: } 161.479 \\
\text { Patients without AHI> } 10 \text { / s: } 26.908 \\
\text { Studies other than Turkish and English: } \\
20.212 \\
\text { Unpublished thesis studies: } 47.089 \\
\text { Reviewes: } 316.200 \\
\text { Conference etc. abstracts, protocols, case } \\
\text { reports, in-vitro studies: } 282.565 \\
\text { Ongoing studies: } 20.181\end{array}$ \\
\hline & $\begin{array}{l}\text { A study that was evaluated for } 4 \text { weeks was } \\
\text { removed. (Staniforth et al. 1998) }\end{array}$ \\
\hline
\end{tabular}

Studies in accordance with the inclusion criteria: 16

Figure 1. Flow Chart

Staniforth et al. (6) European Heart Journal 1998; 19: 922-8.

In the studies, CPAP, BIPAP, ASV, overdrive pacemaker, home oxygen therapy (HOT), slow breathing exercise device (RESPERATE) and structured physical exercise interventions were applied as treatment interventions for the group with SRD (Tables 2, 3). In addition, in the studies evaluated, it was found that symptom evaluation scales such as Epworth Sleepiness Scale (ESS), Pittsburg Sleep Quality Index (PSQI), Fatigue Severity Scale, and disease-specific quality of life scales such as Minnesota Living with HF Questionnaire (MLHFQ) and Chronic HF Questionnaire (Q), and SF-36 general quality of life scale, mental status and motor function assessment tools were used (Table 2).

\section{Results}

The randomized controlled trials included in the study were mostly conducted with male CHF patients who were 60 years of age and over and who had a diagnosis of OSA and CSA (AHI >10/hr., LVEF $\leq 45 \%$ ) (Table 2).

In a study evaluating the effect of CPAP therapy on cardiac functions in CHF patients with OSA, it was reported that CPAP treatment improved LVEF compared to those treated with fake-CPAP (placebo) treatment. However, no significant difference was found in the cardiological variables and quality of life of patients in both the intervention and placebo groups. The authors stated that the improvement in LVEF would not necessarily improve cardiological symptoms (7). However, Khayat et al. (8) stated that, contrary to this study, BIPAP treatment was more effective on improvement in LVEF than CPAP. Bradley et al. (9) reported that CPAP treatment, in addition to medical treatment, improved the CSA and nocturnal oxygenation compared to medical treatment only, and that the CPAP group had more improvement in their functional capacity (six minutes walking distance-6MWD) $(p=0.016)$ (Table 2).

In the study of O'Connor et al. (10), in which the effectiveness of ASV therapy was evaluated, it was found that ASV treatment added to the optimal medical therapy in patients with moderate-to-severe sleep apnea did not improve cardiovascular (CV) outcomes over 6 months, and that functional capacity in both control and intervention group did not differ. However, Arzt et al. (11) reported that ASV treatment added to medical therapy was an effective treatment for CSA and OSA, and that it improved cardiac function compared to medical therapy only in patients with sleep apnea. Similarly, in another study conducted in 2012, it was found that ASV significantly reduced central, periodical various respiratory disorders (12). In another study, it was found that ASV treatment at home caused a mild improvement in sleep fragmentation and improved sleep efficiency in CHF patients with CSA or OSA (13). However, Cowie et al. (14) stated that ASV treatment in addition to medical therapy did not improve outcomes, increased risk of CV death, and had no beneficial effect on quality of life and HF symptoms (Table 2).

Kasai et al. (15) found a significant increase in functional capacity (6MWD) in the ASV-mode group compared to the CPAP-mode group. Priefert et al. (16) found that ASV therapy for patients with $E F<40 \%$ lower ejection fraction (HFrEF) and SRD provided significant improvement in AHI at 12 weeks compared to the group treated with medical therapy only. In the same study, it was found that ASV treatment had an effect on nocturnal ventricular and supraventricular arrhythmias and the number of ventricular beats was less in the ASV treated group compared to the control group (Table 2).

Kawecha-Jaszcz et al. (17) found that the use of slow breathing device at home in patients with stable chronic systolic HF tended to reduce sleep disturbance and predominantly narrow central apnea, improve functional capacity and systolic left ventricular function. In one study, the authors stated that CPAP treatment titrated automatically at night in patients with OSA and CHF improved the daytime sleepiness, but did not improve other quality of life measures or severe CHF markers (Table 2) (18).

In another study, daytime sleepiness was found to be better in the CPAP group than the BIPAP group. In the same study, quality of life, functional capacity and blood pressure (BP) changes were found to be better in the BIPAP group than in CPAP (8). Similarly, Egea et al. (7) reported that CPAP therapy did not show a significant improvement in cardiological changes and quality of life except daytime sleepiness (Table 2).

Nakao et al. (19) reported that nocturnal oxygen therapy for 12 weeks at home improved SRD and had a positive effect on functional capacity in chronic HF patients with CSA (Table 2).

Suna et al. (20) found that exercise training administered to the intervention group significantly improved poor sleep quality in patients with HF followed by a disease management program for 12 weeks (Table 2).

As a result of the studies, only one RCT evaluated the effect of atrial overdrive pacemaker treatment on SRD in systolic HF patients with OSA and the intervention was found to reduce AHI safely (Table 2) (21). 
Table 2. Characteristics of the studies included in the systematic review

\begin{tabular}{|c|c|c|c|c|c|c|c|}
\hline $\begin{array}{l}\text { Source } \\
\text { and sleep } \\
\text { respiratory } \\
\text { disorder type }\end{array}$ & $\begin{array}{l}\text { Method- } \\
\text { randomization }\end{array}$ & Gender-sampling & Duration & $\begin{array}{l}\text { Other evaluation } \\
\text { tools }\end{array}$ & Age & Findings & Results \\
\hline $\begin{array}{l}\text { Egea et al. (7) } \\
\text { OSA }\end{array}$ & $\begin{array}{l}\text { - RCT, multi-center, } \\
\text { - Plasebo ( } \\
\text { fake-CPAP) }(n=32) \\
\text { - CPAP }(n=28)\end{array}$ & $\begin{array}{l}\text { - Mostly male } \\
\text { - A total of } 60 \\
\text { patients } \\
\text { - OSA (AHI >10/h) } \\
\text { - CHF (LVEF <45\%) }\end{array}$ & 3 months & $\begin{array}{l}\text { - ESS } \\
\text { - SF-36 } \\
\text { - NYHA } \\
\text { classification } \\
\text { - Borg dyspnea } \\
\text { scale } \\
\text { - 6MWT }\end{array}$ & $\begin{array}{l}\text { CPAP: } 64 \\
\text { Fake-CPAP: } 63 \\
\text { (mean) }\end{array}$ & $\begin{array}{l}\text { - A } 30 \% \text { improvement in } \\
\text { LVEF compared to baseline } \\
\text { in the CPAP group, less } \\
\text { than } 30 \% \text { improvement in } \\
\text { placebo. } \\
\text { - In contrast to the } \\
\text { placebo group, significant } \\
\text { improvement in } \mathrm{AHI} \\
(\mathrm{p}<0.001) \text { and } \mathrm{SaO}_{2} \\
(\mathrm{p}=0.002) \text { in the CPAP } \\
\text { group }\end{array}$ & $\begin{array}{l}\text { - CPAP treatment } \\
\text { has been shown to } \\
\text { be beneficial in CHF } \\
\text { patients with SRD. } \\
\text { - No difference was } \\
\text { found between the } \\
\text { two groups except } \\
\text { for ESS index in the } \\
\text { cardiac variables } \\
\text { and quality of life } \\
\text { tests }\end{array}$ \\
\hline $\begin{array}{l}\text { Khayat et } \\
\text { al. (8) } \\
\text { OSA }\end{array}$ & $\begin{array}{l}\text { - RCT pilot study, } \\
\text { - } \operatorname{BIPAP}(n=13) \\
\text { CPAP }(n=11)\end{array}$ & $\begin{array}{l}\text { - Female and male } \\
\text { mixed group } \\
\text { - Total } 24 \text { patients } \\
\text { - Stable LV systolic } \\
\text { dysfunction } \\
\text { - NYHA class II-III, } \\
\text { - CHF (LVEF LV 45\%) } \\
\text { - Not hospitalized } \\
\text { in the last } 3 \\
\text { months and } \\
\text { medication was } \\
\text { unchanged. } \\
\text { - New OSA }\end{array}$ & 3 months & $\begin{array}{l}\text { - ESS } \\
\text { - 6MWT } \\
\text { - MLHFQ } \\
\text { - Weekly device } \\
\text { memory check } \\
\text { for treatment } \\
\text { compliance } \\
\text { assessment }\end{array}$ & $\begin{array}{l}\mathrm{BIPAP}=51.3 \\
\mathrm{CPAP}=54.8 \\
\text { (mean) }\end{array}$ & $\begin{array}{l}- \text { AHI was not changed in } \\
\text { both groups ( } p=0.24) \text {. } \\
\text { - Compliance with the } \\
\text { treatment device is slightly } \\
\text { higher in the BIPAP group. } \\
\text { In the CPAP group, the ESS } \\
\text { score was higher ( } p=0.38 \text { ). } \\
\text { Heart rate changes in } \\
\text { CPAP group more effective } \\
\text { ( } p=0.42 \text { ). } \\
\text { - MLHF, } 6 \text { MWD, SBP and } \\
\text { DBP improved in the } \\
\text { BIPAP group }\end{array}$ & $\begin{array}{l}\text { BIPAP treatment } \\
\text { was found to be } \\
\text { more effective } \\
\text { than CPAP in the } \\
\text { treatment of LVEF } \\
\text { in patients with } \\
\text { LV dysfunction } \\
\text { and OSA. Further } \\
\text { studies have } \\
\text { been proposed } \\
\text { to evaluate the } \\
\text { mechanisms } \\
\text { behind this effect }\end{array}$ \\
\hline $\begin{array}{l}\text { Smith et al. } \\
\text { (18) } \\
\text { OSA }\end{array}$ & $\begin{array}{l}\text { RCT, double blind, } \\
\text { placebo control, } \\
\text { diagonal design. } \\
\text { - Automatically } \\
\text { adjusted CPAP (at } \\
\text { least } 6 \text { hours per } \\
\text { night) } \\
\text { - CPAP }(n=12) \\
\text { - Fake-CPAP }(n=11) \\
\text { (placebo) }(1: 1)\end{array}$ & $\begin{array}{l}\text { - Mainly male } \\
\text { - Total } 23 \text { patients } \\
\text { - NYHA class II-IV } \\
\text { - Stable, } \\
\text { symptomatic CHF } \\
\text { (LVEF }<45 \%) \\
\text { - OSA (AHI } \geq 15 / h)\end{array}$ & 6 weeks & $\begin{array}{l}\text { - Clinical } \\
\text { evaluation } \\
\text { - TTE } \\
\text { - CPET } \\
\text { - 6MWT } \\
\text { - Neurohormonal } \\
\text { markers } \\
\text { - OSLER test } \\
\text { - ESS } \\
\text { - Quality of Life } \\
\text { Assessment }\end{array}$ & 61 (mean) & $\begin{array}{l}\text { - In case of daily sleepiness } \\
\text { with CPAP, there was no } \\
\text { objective improvement } \\
\text { (OSES } p=0.63 \text { ), there was } \\
\text { improvement in subjective } \\
\text { assessment (ESS) ( } p=0.04 \text { ). } \\
\text { No significant difference } \\
\text { was found between CPAP } \\
\text { treatment and fake-CPAP } \\
\text { in the evaluation of -LVEF, } \\
\text { exercise capacity and } \\
\text { quality of life }(p>0.05)\end{array}$ & $\begin{array}{l}\text { - CPAP treatment } \\
\text { improved } \\
\text { subjective daily } \\
\text { sleepiness, but } \\
\text { did not improve } \\
\text { other quality of } \\
\text { life measures } \\
\text { or CHF markers. } \\
\text { The symptomatic } \\
\text { benefit in this } \\
\text { patient group is } \\
\text { the alleviation of } \\
\text { OSA rather than the } \\
\text { improvement in } \\
\text { cardiac function }\end{array}$ \\
\hline $\begin{array}{l}\text { Randerath et } \\
\text { al. (12) } \\
\text { OSA } \\
\text { CSA }\end{array}$ & $\begin{array}{l}\text { - RCT, parallel } \\
\text { group, double } \\
\text { blind, single center } \\
\text { clinical study. } \\
\text { - CPAP }(n=34) \\
\text { or ASV }(n=36)+ \\
\text { optimal medical } \\
\text { treatment } \\
\text { - Optimal medical } \\
\text { treatment }\end{array}$ & $\begin{array}{l}\text { - Mainly male } \\
\text { - CHF (LVEF } \geq 20 \% \text { ) } \\
\text { - NYHA class II and } \\
\text { III-AHI } \geq 15 / h r \\
\text { - Central rate } 80 \% \\
\text { - Obstructive rate } \\
20 \%-50 \%\end{array}$ & 1 year & $\begin{array}{l}\text { - ECO at the } \\
\text { baseline, } 3^{\text {rd }}, 12^{\text {th }} \\
\text { months } \\
\text { - CPET } \\
\text { - proBNP } \\
\text { - MLHFQ } \\
\text { - After } 6 \text { and } 9 \\
\text { months, quality } \\
\text { of life data was } \\
\text { received by } \\
\text { telephone. }\end{array}$ & $\begin{array}{l}\text { CPAP }=67.4 \\
\text { ASV }=65.3 \\
\text { (mean) }\end{array}$ & $\begin{array}{l}\text { - Both methods have } \\
\text { significantly improved } \\
\text { total AHI. } \\
\text { - Approximately 50\% } \\
\text { of patients with CPAP } \\
\text { have decreased central } \\
\text { respiratory disability, and } \\
\text { with ASV treatment, there } \\
\text { is a better reduction in AHI } \\
\text { than CPAP. } \\
\text { - Compared to CPAP } \\
\text { treatment at } 12^{\text {th }} \text { month } \\
\text { follow-up, there was an } \\
\text { improvement in periodic } \\
\text { and different respiratory } \\
\text { disorders and proBNP } \\
\text { levels with ASV treatment }\end{array}$ & $\begin{array}{l}\text { It has been } \\
\text { proven that ASV } \\
\text { (compared to CPAP } \\
\text { in patients with } \\
\text { mild to moderate } \\
\text { HF during a } \\
12 \text {-month period) } \\
\text { is effective in } \\
\text { suppressing central, } \\
\text { periodic, different } \\
\text { respiratory } \\
\text { disorders more } \\
\text { effectively and } \\
\text { obstructive events } \\
\text { in an equally } \\
\text { effective manner. } \\
\text { Patients showed } \\
\text { improvement in } \\
\text { BNP levels }\end{array}$ \\
\hline
\end{tabular}




\section{Table 2 contiuned}

\begin{tabular}{|c|c|c|c|c|c|c|c|}
\hline $\begin{array}{l}\text { Kasai et al. } \\
\text { (15) } \\
\text { CSA }\end{array}$ & $\begin{array}{l}\text { - RCT, prospective, } \\
\text { single-center, } \\
\text { single-blind study. } \\
\text { - CPAP mode or ASV } \\
\text { mode }\end{array}$ & $\begin{array}{l}\text { - Gender not } \\
\text { specified. } \\
\text { - Total } 74 \text { patients } \\
\text { - NYHA } \geq 1 \text { I } \\
\text { - CHF (LVEF <50\%) } \\
\text { - AHI } \geq 15 / h\end{array}$ & 3 months & $\begin{array}{l}\text { - PSG } \\
\text { - BMI } \\
\text { - ABG } \\
\text { - CV variables } \\
\text { - 6MWT } \\
\text { - ECHO } \\
\text { - LVEF } \\
\text { - SF-36 quality of } \\
\text { life assessment }\end{array}$ & $\begin{array}{l}\text { CPAP: } 65.8 \\
\text { ASV }=64.3 \\
\text { (mean) }\end{array}$ & $\begin{array}{l}\text { - Significant reduction in } \\
\text { plasma proBNP level in } \\
\text { the ASV mode group. } \\
\text { The decrease in UNE and } \\
\text { increase in 6MWD are } \\
\text { significantly higher in the } \\
\text { ASV group. } \\
\text { Significant reduction in LV } \\
\text { end-systolic diameters in } \\
\text { the LV group }\end{array}$ & $\begin{array}{l}\text { ASV treatment has } \\
\text { been shown to } \\
\text { improve underlying } \\
\text { dysfunction in non- } \\
\text { CPAP-induced CSA } \\
\text { Japanese male HF } \\
\text { patients. Effective } \\
\text { suppression of CSA } \\
\text { has been shown } \\
\text { to be useful in } \\
\text { improving cardiac } \\
\text { function. It is } \\
\text { recommended to } \\
\text { investigate the } \\
\text { long-term effects } \\
\text { of CSA treatment } \\
\text { with ASV }\end{array}$ \\
\hline $\begin{array}{l}\text { Bradley et } \\
\text { al. (9) } \\
\text { CSA }\end{array}$ & $\begin{array}{l}\text { RCT, open-label, } \\
\text { multi-center } \\
\text { (11-center) } \\
\text { operation. } \\
\text { - CPAP + optimal } \\
\text { medical treatment } \\
\text { ( } n=128) \\
\text { - Optimal medical } \\
\text { treatment ( } n=130 \text { ) } \\
\text { at least } 6 \text { hours of } \\
\text { CPAP use at home } \\
\text { during the night }\end{array}$ & $\begin{array}{l}\text { - Mainly male } \\
\text { Total of } 258 \\
\text { patients } \\
\text { - NYHA class II-IV } \\
\text { - At least one } \\
\text { medical treatment } \\
\text { - Stable CHF (LVEF } \\
<40 \% \text { ) } \\
\text { - CSA }\end{array}$ & 3 months & $\begin{array}{l}\text { - Radionuclide } \\
\text { angiography } \\
\text { resting LVEF } \\
\text { - 6MWT } \\
\text { - Life Quality } \\
\text { Survey } \\
\text { Atrial natriuretic } \\
\text { peptide } \\
\text { and plasma } \\
\text { norepinephrine } \\
\text { levels were } \\
\text { measured at -3 } \\
\text { centers }\end{array}$ & 18-79 & $\begin{array}{l}\text { In the CPAP group, } \\
6 \text { MWD increased more at } \\
\text { three-month follow-up } \\
\text { ( } p=0.016) \text {. } \\
\text { There is no significant } \\
\text { difference between } \\
\text { the two groups in the } \\
\text { Chronic Heart Failure } \\
\text { Questionnaire } \\
\text { A significant increase in } \\
\text { LVEF in the CPAP group } \\
\text { ( } p=0.02 \text { ). } \\
\text { - Plasma norepinephrine } \\
\text { level decreased } \\
\text { significantly ( } p=0.009)\end{array}$ & $\begin{array}{l}\text { - CPAP improved } \\
\text { central sleep } \\
\text { apnea, nocturnal } \\
\text { oxygenation, } \\
\text { left ventricular } \\
\text { function, } \\
\text { sympathetic } \\
\text { nerve activity, } \\
\text { and submaximal } \\
\text { exercise } \\
\text { performance. } \\
\text { - The CANPAP study } \\
\text { did not show a } \\
\text { beneficial effect of } \\
\text { CPAP on morbidity } \\
\text { or mortality in } \\
\text { patients with } \\
\text { central sleep } \\
\text { apnea and heart } \\
\text { failure. There was } \\
\text { no significant } \\
\text { difference in } \\
\text { mortality rates } \\
\text { between the two } \\
\text { groups }\end{array}$ \\
\hline $\begin{array}{l}\text { Hetzenecker } \\
\text { et al. (13) } \\
\text { OSA } \\
\text { CSA }\end{array}$ & $\begin{array}{l}\text { - RCT, multi-center, } \\
\text { parallel group } \\
\text { - ASV (BIPAP-ASV) + } \\
\text { medical treatment } \\
(n=32) \\
\text { - Optimal medical } \\
\text { treatment ( } n=31) \\
(1: 1)\end{array}$ & $\begin{array}{l}\text { - Mainly male } \\
\text { - Total } 63 \text { patients } \\
\text { - Severe sleep } \\
\text { apnea } \\
\text { - CHF }\end{array}$ & 12 weeks & $\begin{array}{l}\text { - Actigraphy device } \\
\text { (wrist) } \\
\text { - BMI } \\
\text { - Resting heart rate } \\
\text { - NYHA } \\
\text { - proBNP }\end{array}$ & $\begin{array}{l}-\mathrm{ASV}=64 \\
- \text { Control=65 } \\
\text { (mean) }\end{array}$ & $\begin{array}{l}\text { - Significant greater } \\
\text { reduction in sleep } \\
\text { fragmentation index } \\
\text { compared to the control } \\
\text { group and significantly } \\
\text { improved sleep efficiency } \\
\text { and proBNP decrease in } \\
\text { the ASV group }(p=0.062)\end{array}$ & $\begin{array}{l}\text { The study showed } \\
\text { that ASV treatment } \\
\text { at home could } \\
\text { reduce sleep } \\
\text { disruption and } \\
\text { improve sleep } \\
\text { quality in CHF } \\
\text { patients with severe } \\
\text { CSA or OSA }\end{array}$ \\
\hline $\begin{array}{l}\text { O'Connor et } \\
\text { al. (10) } \\
\text { OSA }\end{array}$ & $\begin{array}{l}\text { - RCT, multi-center } \\
\text { clinical study } \\
\text { - ASV } \\
\text { - General care } \\
\text { control group (1:1) }\end{array}$ & $\begin{array}{l}\text { - Mostly male } \\
\text { - Reduced or } \\
\text { preserved EF } \\
\text { - AHI } \geq 15 / \mathrm{h} \\
\text { - Hospitalized HF } \\
\text { patients }\end{array}$ & 6 months & $\begin{array}{l}\text { - BMI } \\
\text { - NYHA } \\
\text { - LVEF } \\
\text { - proBNP } \\
\text { - ESS } \\
\text { - SaO2 } \\
\text { - 6MWT }\end{array}$ & 62 (mean) & $\begin{array}{l}\text { - There was a significant } \\
\text { decrease in baseline } \\
\text { in both groups in AHI } \\
\text { ( } p=0.0001) \text {. } \\
\text { - 6MWD was similar in } \\
\text { both groups ( } p>0.05)\end{array}$ & $\begin{array}{l}\text { It was found that } \\
\text { ASV treatment, } \\
\text { which was added } \\
\text { to the optimal } \\
\text { medical treatment } \\
\text { compared to those } \\
\text { receiving only } \\
\text { optimal medical } \\
\text { treatment, did } \\
\text { not improve CV } \\
\text { outcomes over a } \\
\text { 6-month period. }\end{array}$ \\
\hline
\end{tabular}


Table 2 contiuned

\begin{tabular}{|c|c|c|c|c|c|c|c|}
\hline $\begin{array}{l}\text { Cowie et al. } \\
\text { (14) } \\
\text { CSA }\end{array}$ & $\begin{array}{l}\text { - RCT, Multi- } \\
\text { center, parallel } \\
\text { group, event- } \\
\text { based study. } \\
\text { - ASV + medical } \\
\text { treatment } \\
\text { ( } n=666) \\
\text { - Medical } \\
\text { treatment } \\
(n=659)\end{array}$ & $\begin{array}{l}\text { - Mainly male } \\
\text { - Symptomatic } \\
\text { CHF (LVEF CHF } \\
45 \% \text { ) } \\
\text { - Hospitalized } \\
\text { at least } 1 \text { time } \\
\text { within } 2 \text { years, } \\
\text { - NYHA II, III or IV } \\
\text { - CSA (AHI } \geq 15 / h \text {, } \\
>50 \% \text { of apnea or } \\
\text { hypopnea event } \\
\text { and central AHI } \\
\geq 10 / \mathrm{hr} \text { ) }\end{array}$ & $\begin{array}{l}\text {-Evaluation } \\
\text { on first } \\
2 \text { weeks, } \\
3 \text { and } 12 \\
\text { months } \\
\text { - On the } 6^{\text {th }} \\
\text { and } 12^{\text {th }} \\
\text { months, } \\
\text { phone } \\
\text { contact. } \\
\text {-Total } 31 \\
\text { months } \\
\text { follow-up }\end{array}$ & $\begin{array}{l}\text { - NYHA } \\
\text { classification } \\
\text { - 6MWT } \\
\text { - EQ-5D } \\
\text { - MLHFQ } \\
\text { - ESS }\end{array}$ & 69 (mean) & $\begin{array}{l}\text { - SRD was better } \\
\text { controlled during ASV } \\
\text { treatment. } \\
\text { - AHI 6,6/h, ODD 8,6/h } \\
\text { - Mortality and CV } \\
\text { mortality for all } \\
\text { causes were higher } \\
(29.3 \%, 24.0 \% \text { ) than } \\
\text { in the control group, } \\
\text { respectively ( } 34.8 \% \text {, } \\
29.9 \%) \text { in the ASV group } \\
(p<0.05)\end{array}$ & $\begin{array}{l}\text { - In addition to } \\
\text { guideline-based } \\
\text { medical therapy, } \\
\text { the treatment of } \\
\text { ASV was found } \\
\text { not to improve } \\
\text { results. } \\
\text { - It was found that } \\
\text { ASV increased the } \\
\text { risk of CV death } \\
\text { (34\%) and had no } \\
\text { beneficial effect } \\
\text { on quality of life } \\
\text { or HF symptoms. } \\
\text { These results } \\
\text { were seen despite } \\
\text { effective control } \\
\text { of central sleep } \\
\text { apnea during } \\
\text { treatment of ASV }\end{array}$ \\
\hline $\begin{array}{l}\text { Arzt et al. } \\
(11) \\
\text { OSA } \\
\text { CSA }\end{array}$ & $\begin{array}{l} \\
\text { - RCT } \\
\text { - ASV (BIPAP) + } \\
\text { optimal medical } \\
\text { treatment ( } n=37) \\
\text { - Optimal medical } \\
\text { treatment ( } n=35) \\
(1: 1)\end{array}$ & $\begin{array}{l} \\
\text { - Mostly male } \\
\text { - Total } 72 \\
\text { - Stable CHF (LVEF) } \\
40 \% \text { (in the last } 4 \\
\text { weeks) } \\
\text { - NYHA class II-III } \\
\text { - AHI } \geq 20 / \text { hr }\end{array}$ & 12 weeks & $\begin{array}{l} \\
\text { - SF-36 } \\
\text { - MLHFQ } \\
\text { - proBNP } \\
\text { - Creatinine } \\
\text { - GFR } \\
\text { - Fatigue severity } \\
\text { scale } \\
\text { - ESS }\end{array}$ & $\begin{array}{l}-\mathrm{ASV}=64 \\
- \text { Control=65 } \\
\text { (mean) }\end{array}$ & $\begin{array}{l}\text { - Moderate improvement } \\
\text { in LVEF in both groups } \\
(p>0.05) \text {. } \\
\text { - In the ASV group, } \\
\text { AHI and central AHI } \\
\text { significantly decreased } \\
(p<0.001) \text {. } \\
\text { - Significant increase in } \\
\text { mean arterial SaO in the } \\
\text { ASV group ( } p=0.001) \text {. } \\
\text { - Further decline in BNP } \\
\text { in the ASV group, and an } \\
\text { increase in GFR ( } p>0.05 \text { ). } \\
\text { - Quality of life and } \\
\text { symptom scores were } \\
\text { similar in both groups } \\
\text { ( } p>0.05 \text { ) }\end{array}$ & $\begin{array}{l}\text { The study } \\
\text { showed that ASV } \\
\text { is an effective } \\
\text { treatment for } \\
\text { both CSA and } \\
\text { OSA in CHF } \\
\text { patients. It has } \\
\text { been shown that } \\
\text { ASV treatment } \\
\text { decreases BNP } \\
\text { levels in CHF } \\
\text { patients with SRD, } \\
\text { thus improving } \\
\text { cardiac functions }\end{array}$ \\
\hline $\begin{array}{l}\text { Lyons et al. } \\
(22) \\
\text { OSA } \\
\text { CSA }\end{array}$ & $\begin{array}{l}\text { - RCT, multi- } \\
\text { center, } \\
\text { multinational, } \\
\text { parallel group, } \\
\text { open label, single } \\
\text { blind } \\
\text { - ASV + standard } \\
\text { medical } \\
\text { treatment } \\
\text { - Standard } \\
\text { medical } \\
\text { treatment } \\
\text { - } 430 \text { OSA and } \\
430 \text { CSA patients }\end{array}$ & $\begin{array}{l}\text { - Men and women } \\
\text { - Stable HFrEF } \\
\text { (LVEF } 345) \text { for at } \\
\text { least } 3 \text { months } \\
\text { - SRD (AHI } \geq 15 / h)\end{array}$ & $\begin{array}{l}\text { In a } \\
\text { maximum } \\
\text { period of } 5 \\
\text { years; basal, } \\
1^{\text {st }}, 3^{\text {rd }} \text { and } \\
6^{\text {th }} \text { months } \\
\text { and every } \\
6 \text { months: } \\
\text { PSG } \\
\text { In } 6 \\
\text { months: } \\
\text { - ECHO } \\
\text { - proBNP } \\
\text { - } 6 \text { MWT }\end{array}$ & $\begin{array}{l}\text { - Physical } \\
\text { examination } \\
\text { - 6MWT } \\
\text { - MLHFQ } \\
\text { - ESS }\end{array}$ & $\begin{array}{l}18 \text { years and } \\
\text { over }\end{array}$ & $\begin{array}{l}\text { - OSA in HFrEF patients is } \\
\text { more common than CSA. } \\
\text { - ASVmV improves the } \\
\text { CSA, but there is no } \\
\text { difference in the first } \\
\text { evaluation between } \\
\text { those who are not using } \\
\text { ASV mode. } \\
\text { - Secondary evaluation } \\
\text { of the ASV mode for all } \\
\text { causes and increased } \\
\text { mortality and morbidity } \\
\text { in CV }\end{array}$ & $\begin{array}{l}\text { According to the } \\
\text { results of the } \\
\text { ADVENT-HF study, } \\
\text { data are provided } \\
\text { to improve the } \\
\text { quality of life } \\
\text { and decrease } \\
\text { the morbidity } \\
\text { and mortality in } \\
\text { patients with OSA } \\
\text { and HFrEF treated } \\
\text { with ASV. } \\
\text { The information } \\
\text { obtained from } \\
\text { the ADVENT- } \\
\text { HF study will } \\
\text { provide important } \\
\text { information in } \\
\text { the treatment } \\
\text { of patients with } \\
\text { HFREF and CSA } \\
\text { in the SERVE-HF } \\
\text { study }\end{array}$ \\
\hline
\end{tabular}




\section{Table 2 contiuned}

\begin{tabular}{|c|c|c|c|c|c|c|c|}
\hline $\begin{array}{l}\text { Priefert, et al. } \\
(16) \\
\text { OSA } \\
\text { CSA }\end{array}$ & $\begin{array}{l}\text { - RCT, multi-center, } \\
\text { controlled, parallel, } \\
\text { open label. } \\
\text { - ASV (BIPAP-ASV) } \\
\text { + optimal medical } \\
\text { treatment at night } \\
\text { ( } n=37) \\
\text { - Optimal medical } \\
\text { treatment }(n=35)\end{array}$ & $\begin{array}{l}\text { - Mostly male } \\
\text { - A total of } 72 \\
\text { patients } \\
\text { - Stable HFrEF } \\
\text { - SRD }\end{array}$ & 12 weeks & $\begin{array}{l}\text { - PSG } \\
\text { - NYHA } \\
\text { - Pulse } \\
\text { - LVEF } \\
\text { - BP } \\
\text { - BMI } \\
\text { - EEG } \\
\text { - EOG } \\
\text { - EMG } \\
\text { - SaO } \\
\text { - Single channel } \\
\text { ECG }\end{array}$ & $\begin{array}{l}\text { - Control=67 } \\
\text { - ASV=65 (mean) }\end{array}$ & $\begin{array}{l}\text { - Significant improvement } \\
\text { in AHI/hr. }(p<0.001) \text {, } \\
\text { obstructive AHI/hr. } \\
(p=0.003) \text { and central AHI/ } \\
\text { hr. }(p<0.001) \text { in ASV group. } \\
\text { - Further improvement } \\
\text { in total sleep time (min.) } \\
(p=0.259) \text { and mean } \mathrm{SaO}_{2} \\
(\%)(p=0.0031) \text { in the ASV } \\
\text { group. } \\
\text { - The number of single, } \\
\text { multiple and double VEAs } \\
\text { in ASV group was less } \\
\text { ( } p>0.05) \text {. } \\
\text { - Decreased heart rate } \\
\text { (min) in the treatment } \\
\text { of ASV, but no significant } \\
\text { difference between the } \\
\text { groups ( } p>0.05 \text { ) }\end{array}$ & $\begin{array}{l}\text { In the study, it } \\
\text { was stated that } \\
\text { ASR treatment } \\
\text { could be applied } \\
\text { in patients with } \\
\text { HFrEF and SRD. It } \\
\text { is recommended } \\
\text { to repeat the study } \\
\text { with larger patient } \\
\text { groups }\end{array}$ \\
\hline $\begin{array}{l}\text { Nokao et al. } \\
(19) \\
\text { CSA }\end{array}$ & $\begin{array}{l}\text { - RCT, open label, } \\
\text { multi-center } \\
\text { operation. } \\
\text { - HOT ( } 3 \text { liters } \\
\text { oxygen therapy per } \\
\text { minute (with 92\% } \\
\text { density) with nasal } \\
\text { cannula + optimal } \\
\text { medical treatment } \\
\text { - Optimal medical } \\
\text { treatment }\end{array}$ & $\begin{array}{l}\text { - Mostly male } \\
\text { - Short term: } 30 \\
\text { HOT, } 33 \text { controls in } \\
\text { total } 63 \\
\text { - Long term: } 26 \\
\text { HOT, } 25 \text { controls } \\
\text { total } 51 \\
\text { - A total of } 107 \\
\text { patients } \\
\text { - CSA } \\
\text { - Symptomatic, } \\
\text { stable CHF (LVEF, } \\
45 \%) \\
\text { - NYHA II-III } \\
\text { - At least } 5 \text { episodes } \\
\text { of apnea-hypopnea } \\
\text { with } \geq 5 / \text { hr. } \\
\text { reduction in ODI } \\
\text { and } 5 \text { hours of } \\
\text { sleep }\end{array}$ & $\begin{array}{l}\text { Baseline } \\
\text { and 12-week } \\
\text { evaluation } \\
\text { with two- } \\
\text { channel } \\
\text { real-time } \\
\text { Holter device } \\
\text { at night }\end{array}$ & $\begin{array}{l}\text { - PSG } \\
\text { - NYHA } \\
\text { classification } \\
\text { - Pulse } \\
\text { - LVEF } \\
\text { - proBNP } \\
\text { - } \mathrm{SaO}_{2} \\
\text { - Specific Activity } \\
\text { Scale (Mets) } \\
\text { - QoL scale }\end{array}$ & $\begin{array}{l}\text { - } \mathrm{HOT}=65.3 \\
\text { - Control=66.5 } \\
\text { (mean) }\end{array}$ & $\begin{array}{l}\text { - Significant improvement } \\
\text { in AHI ( } p<0.01) \text {, a } \\
\text { significant decrease in CAI } \\
(p<0.05) \text { in the HOT group. } \\
\text { - Significant improvement } \\
\text { in ODI ( } p<0.01) \text {, increase } \\
\text { in functional capacity } \\
(p<0.01) \text {, further } \\
\text { improvement in LVEF } \\
\text { ( } p>0.05) \text {, greater reduction } \\
\text { in VEAs ( } p>0.05) \text {, further } \\
\text { improvement in NYHA } \\
(p=0.02) \text { in the HOT group }\end{array}$ & $\begin{array}{l}\text { - HOT has been } \\
\text { shown to improve } \\
\text { SRD, quality of } \\
\text { life, and cardiac } \\
\text { function in patients } \\
\text { with CHF and CSA. } \\
\text { The efficacy of } \\
\text { HOT on ventricular } \\
\text { arrhythmias } \\
\text { has not been } \\
\text { demonstrated. } \\
\text { Further studies } \\
\text { have been } \\
\text { proposed }\end{array}$ \\
\hline $\begin{array}{l}\text { Kawechka- } \\
\text { Jaszcz et al. } \\
\text { (17) } \\
\text { CSA }\end{array}$ & $\begin{array}{l}\text { - RCT, cross design, } \\
\text { open label } \\
\text { - Slow Respiratory } \\
\text { Exercise (SRE) with } \\
\text { + RESPERATE + } \\
\text { optimal medical } \\
\text { treatment } \\
\text { - The standard care } \\
\text { group }\end{array}$ & $\begin{array}{l}\text { - } 86 \text { male, } 24 \\
\text { female with a } \\
\text { total of } 110 \text { stable } \\
\text { HF patients (LVEF } \\
<40 \% \text { ). } \\
\text { - NYHA class I-III } \\
\text { - A 24-hour Holter } \\
\text { with sinus rhythm } \\
\text { - Those who have } \\
\text { the ability to apply } \\
\text { breathing exercises } \\
\text { after training }\end{array}$ & $10-12$ weeks & $\begin{array}{l}\text { - PSG } \\
\text { - Ambulatory } \\
\text { cardiorespiratory } \\
\text { device (Emblatta } \\
\text { Gold) } \\
\text { - ECHO } \\
\text { - 6MWT } \\
\text { - NYHA } \\
\text { - BP } \\
\text { - BMI } \\
\text { - Laboratory tests }\end{array}$ & $\begin{array}{l}23-87 \\
64.5 \text { (mean) }\end{array}$ & $\begin{array}{l}\text { - Significant decrease } \\
\text { in global AHI in the SRE } \\
\text { group }(p=0.043) \text {, } \\
\text { - Significant increase in } \\
\text { LVEF }(p=0.03) \text { and 6MWD } \\
(p<0.001)\end{array}$ & $\begin{array}{l}\text { - In stable systolic } \\
\text { CHF patients, } \\
\text { SRE was found to } \\
\text { reduce narrow } \\
\text { central apnea } \\
\text { and hypopnea in } \\
\text { SRD, improving } \\
\text { functional capacity } \\
\text { and systolic LVEF. } \\
\text { It is stated that the } \\
\text { SRE device can be } \\
\text { used successfully } \\
\text { as a home-based } \\
\text { rehabilitation tool } \\
\text { in CHF patients }\end{array}$ \\
\hline
\end{tabular}


Table 2 contiuned

\begin{tabular}{|c|c|c|c|c|c|c|c|}
\hline $\begin{array}{l}\text { Sharafkhaneh } \\
\text { et al. (21) } \\
\text { OSA }\end{array}$ & $\begin{array}{l}\text { - RCT } \\
\text { - Atrial overdrive } \\
\text { pacemaker } \\
\text { - CPAP }\end{array}$ & $\begin{array}{l}\text { - } 15 \text { men } \\
\text { - Medium- } \\
\text { severe OSA with } \\
\text { pacemaker applied } \\
46 \text { months ago } \\
\text { - Stable systolic HF } \\
\text { (LVEF <55\%) }\end{array}$ & $\begin{array}{l}\text { Pacemaker } \\
\text { implanted } \\
46 \text { months } \\
\text { before the } \\
\text { study }\end{array}$ & - ESS & 74 (mean) & $\begin{array}{l}\text { - Decrease in } \mathrm{AHI}(7 \pm 7.9) \\
\text { and total stimulation } \\
\text { index }(17 \pm 16.8) \text { with CPAP } \\
\text { treatment. } \\
\text { - Improvement at } \\
\text { nocturnal } \mathrm{SaO}_{2}\end{array}$ & $\begin{array}{l}\text { - Atrial overdrive } \\
\text { pacemaker has } \\
\text { been shown to } \\
\text { reduce AHI safely. } \\
\text { - Although } \\
\text { atrial overdrive } \\
\text { pacemaker is not } \\
\text { as effective as CPAP } \\
\text { in the treatment } \\
\text { of OSA, it may play } \\
\text { a therapeutic role } \\
\text { in patients with } \\
\text { systolic cardiac } \\
\text { dysfunction with } \\
\text { other treatment } \\
\text { modalities }\end{array}$ \\
\hline $\begin{array}{l}\text { Suna et al. } \\
(20) \\
\text { OSA } \\
\text { CSA }\end{array}$ & $\begin{array}{l}\text { - RCT (sub-study) } \\
\text { - Disease training } \\
\text { program + } \\
\text { structured physical } \\
\text { exercise training } \\
\text { ( } \mathrm{n}=54 \text { ) } \\
\text { - Self-management } \\
\text { support program } \\
\text { (+52), which } \\
\text { includes disease } \\
\text { training program + } \\
\text { standard exercise } \\
\text { recommendations }\end{array}$ & $\begin{array}{l}\text { - Mostly male } \\
\text { - A total of } 106 \\
\text { patients }\end{array}$ & 12 weeks & $\begin{array}{l}\text { - PSQI } \\
\text { - 6MWT } \\
\text { - GDS } \\
\text { - BMI } \\
\text { - Resting heart rate }\end{array}$ & $\begin{array}{l}\text { - Control=62 } \\
\text { - Intervention=61 } \\
\text { (mean) }\end{array}$ & $\begin{array}{l}\text { - Significant improvement } \\
\text { in sleep disturbance } \\
(p<0.01) \text {, sleep duration, } \\
\text { drowsiness during the } \\
\text { day, sleep efficiency } \\
\text { habit scores were not } \\
\text { significantly different in } \\
\text { the intervention group. } \\
\text { - PSQI-Subjective sleep } \\
\text { quality and global scores } \\
\text { significantly improved in } \\
\text { the intervention group } \\
(p<0.01)\end{array}$ & $\begin{array}{l}\text { In the second week } \\
\text { of } 12 \text {-week follow- } \\
\text { up, heart failure } \\
\text { patients visited } \\
\text { by the exercise } \\
\text { physiologist were } \\
\text { found to have } \\
\text { improved sleep } \\
\text { quality }\end{array}$ \\
\hline
\end{tabular}

\section{Effect of Adaptive Servo-ventilation Methods and Continuous Positive Airway Pressure on Mortality}

Two studies published in 2015 showed that there was no significant difference between the ASV treatment group and the medical treatment group in terms of quality of life or changes in HF symptoms (14), and that ASV treatment increased the risk of death due to all reasons and CV death $(p<0.05)(14)$. In contrast to these studies, the ADVENT-HF study showed that treatment with ASV in HFrEF patients improved health-related quality of life and reduced morbidity and mortality (22) (Tables 2, 3).

\section{Discussion}

One newly defined factor that is considered to contribute to morbidity and mortality in CHF is SRD. Sleep respiratory disorder is usually defined as OSA and CSA (23).

As a result of the polysomnographic examination, the presence of more than 5 AHIs indicates the presence of OSA. For the diagnosis of OSA with AHI, excessive daytime sleepiness and cardiac disorders are expected. It has been shown that the risk of morbidity and mortality increases especially when $\mathrm{AHI}$ is more than 20 (2).

Non-invasive mechanical ventilator devices used in the treatment of PAP include CPAP, Auto CPAP (APAP), BIPAP, Auto BIPAP, BIPAP-ST (/Auto), volume cycle ventilator (AVAPS/IVAPS), and Servo-Ventilator (/Auto). The first treatment option for the treatment of SRD is CPAP. The aim of the treatment of SRD is to ensure that the upper airway remains open during sleep and to regulate ventilation and sleep quality (2). This method has been shown to significantly improve symptoms such as snoring, morning headaches and daytime sleepiness in many studies. It is stated that BIPAP treatment should be considered for the treatment of CSA in CHF patients to normalize AHI when it does not respond to adequate CPAP, ASV and oxygen treatments. However, it is also emphasized that ASV treatment is indicated for the treatment of CSA (23).

As a result of the evaluations made in this study, treatment interventions for CHF patients with OSA and CSA included mostly CPAP $(7-9,12,18)$ and ASV (10-16,22), as well as BIPAP (8), overdrive pacing (21), oxygen therapy with nasal cannula $(6,19)$, slow breathing training (17) and exercise training (20).

In these studies, the CPAP treatment for the treatment of CHF patients with OSA was found to improve $\operatorname{LVEF}(7)$ and daytime sleepiness $(7,8,18)$, and ASV treatment was also found to improve AHI $(10,11,16)$, reduce obstructive events, improve respiratory distress (12) and quality of life (22), increase oxygen saturation $(11,16)$ and sleep time, reduce ventricular premature stroke and mean heart rate (16) (Tables 2,3). 
Table 3. Treatment interventions for the treatment of sleep respiratory disorders

\begin{tabular}{|c|c|c|c|c|c|c|c|}
\hline $\begin{array}{l}\text { Author and Type of Sleep } \\
\text { Respiratory Disorder }\end{array}$ & CPAP & BIPAP & ASV & PM & $\begin{array}{l}\text { Oxygen } \\
\text { Treatment }\end{array}$ & $\begin{array}{l}\text { Slow } \\
\text { Respiratory } \\
\text { Exercise }\end{array}$ & $\begin{array}{l}\text { Physical } \\
\text { Exercise }\end{array}$ \\
\hline $\begin{array}{l}\text { Egea et al. (7) } \\
\text { (OSA) }\end{array}$ & $\begin{array}{l}\text { - LVEF (+) } \\
\text { - Sleepiness (+) } \\
\text { - QOL (-) } \\
\text { - Functional capacity (-) }\end{array}$ & - & - & - & - & - & - \\
\hline Smith et al. (18) (OSA) & $\begin{array}{l}\text { - Sleepiness (+) } \\
\text { - QOL (-) } \\
\text { - CV markers (-) }\end{array}$ & - & - & - & - & - & - \\
\hline Khayat et al. (8) (OSA) & Sleepiness (+) & $\begin{array}{l}\text { - LVEF (+) } \\
\text { - QOL (+) } \\
\text { - Functional } \\
\text { capacity (+) } \\
\text { - Blood pressure } \\
(+) \\
\text { - AHI/hour (-) }\end{array}$ & - & - & - & - & - \\
\hline Bradley et al. (9) (CSA) & $\begin{array}{l}\text { - } \operatorname{LVEF}(+) \\
\text { - Sympathetic nerve } \\
\text { activity }(+) \\
\text { - Nocturnal } \mathrm{SaO}_{2}(+) \\
\text { - Mortality for all causes (-) }\end{array}$ & - & - & & - & - & - \\
\hline $\begin{array}{l}\text { Hetzenecker et al. (13) } \\
\text { (OSA, CSA) }\end{array}$ & - & Sleep quality (+) & - & - & - & - & - \\
\hline Lyons et al. (22) (OSA) & - & - & $\begin{array}{l}\text { QOL }(+) \\
\text { Mortality for all causes (-) } \\
\text { Morbidity (-) }\end{array}$ & - & - & - & - \\
\hline O'Connor et al. (10) (OSA) & - & - & $\begin{array}{l}\text { AHI (+) } \\
\text { CV symptom (-) } \\
\text { Functional capacity (-) }\end{array}$ & - & - & - & - \\
\hline Cowie et al. (14) (OSA) & - & - & $\begin{array}{l}\text { QOL (-) } \\
\text { CV symptom (-) } \\
\text { CV mortality (-) }\end{array}$ & - & - & - & - \\
\hline $\begin{array}{l}\text { Randerath et al. (12) (OSA, } \\
\text { CSA) }\end{array}$ & & - & $\begin{array}{l}\text { OSA }(+) \\
\text { CSA }(+) \\
\text { NT-proBNP }(+)\end{array}$ & & - & - & \\
\hline Kasai et al. (15) (CSA) & - & - & $\begin{array}{l}\text { NT-proBNP }(+) \\
\text { UNE }(+) \\
\text { Functional capacity (-) } \\
\text { LV end-systolic diameters } \\
(+)\end{array}$ & - & - & - & - \\
\hline $\begin{array}{l}\text { Arzt et al. (11) } \\
\text { (OSA, CSA) }\end{array}$ & - & - & $\begin{array}{l}\text { NT-proBNP (+) } \\
\text { AHI / hour (+) } \\
\text { Mean } \mathrm{SaO}_{2}(+) \\
\text { GFR (+) } \\
\operatorname{LVEF~(-)~} \\
\text { QOL (-) } \\
\text { Symptom scores (-) }\end{array}$ & - & - & - & - \\
\hline
\end{tabular}




\section{Table 3 contiuned}

\begin{tabular}{|c|c|c|c|c|c|c|}
\hline Priefert et al. (16) (OSA, CSA) & ( & $\begin{array}{l}\text { AHI / hour (+) } \\
\text { Obstructive AHI / hour (+) } \\
\text { Central AHI / hour }(+) \\
\text { Sleep time }(+) \\
\text { Mean } \mathrm{SaO}_{2}(+) \\
\text { VEA }(+) \\
\text { Mean heart rate }(+)\end{array}$ & - & - & - & - \\
\hline $\begin{array}{l}\text { Sharafkhaneh et al. (21) } \\
\text { (OSA) }\end{array}$ & - & - & $\begin{array}{l}\text { AHI/hour } \\
(+)\end{array}$ & - & - & - \\
\hline Nokao et al. (19) (CSA) & - & - & - & $\begin{array}{l}\text { CSA (+) } \\
\text { QOL (+) } \\
\text { Functional } \\
\text { capacity (+) } \\
\text { VEA (+) } \\
\text { AHI / hour } \\
(+) \\
\operatorname{LVEF~(+)~}\end{array}$ & - & - \\
\hline $\begin{array}{l}\text { Kawecha-Jaszcz et al. (17) } \\
\text { (OSA, CSA) }\end{array}$ & - & - & - & - & $\begin{array}{l}\text { OSA (+) } \\
\text { CSA (+) } \\
\text { Functional } \\
\text { capacity (+) } \\
\text { Systolic LVEF } \\
(+)\end{array}$ & _ \\
\hline $\begin{array}{l}\text { Suna et al. (20) } \\
\text { (OSA, CSA) }\end{array}$ & - & - & - & - & & $\begin{array}{l}\text { Sleep } \\
\text { quality (+) }\end{array}$ \\
\hline
\end{tabular}

The American College of Cardiology/American Heart Association 2013 guide states that CPAP may be useful for improving functional status and increasing LVEF in HF patients with sleep apnea (Evidence B) (24). Similarly, HF Society of America (2010) guideline also recommends the use of CPAP therapy in HF patients with a diagnosis of PSG-documented OSA to improve daily functional capacity and quality of life (25). The treatment of OSA with CPAP showed decrease in sympathetic flow and BP during sleep. The results of studies describing the effect of long-term CPAP treatment on BP in patients with OSA are not very impressive. Most studies have reported a reduction in systolic or diastolic pressure between 2-10 $\mathrm{mmHg}$ after several weeks of CPAP treatment. These studies have shown that the effect of CPAP treatment on BP in patients with OSA is modest and variable (26).

In patients with HF, CSA appears as CSR, a periodic respiration resulting in a long apnea or hypopnea. CSA, which is quite common in HF, is seen in $30-50 \%$ of patients. CPAP treatment aimed at normalizing $\mathrm{AHI}$ is shown as the first treatment to be considered for CHF patients (23). CPAP treatment intervention for the treatment of HF patients with CSA has been found to improve nocturnal oxygenation, LVEF, sympathetic nerve activity and functional capacity (9), however, in the same patient group, ASV treatment has been found to decrease plasma proBNP $(12,15)$, the amount of norepinephrine in 24-hour urine (UNE), left ventricular end systolic diameter (15), in central $\mathrm{AHI}$ and to increase functional capacity (16). Improvement in LVEF is associated with improvement in plasma BNP levels (Table 2,3) (27). For this reason, BNP levels were also examined in the studies in which ASV and CPAP treatments were used. In contrast, Hastings et al. (27) found no change in BNP levels in ASVtreated patients compared to the control group.

Adaptive servo-ventilators work primarily with the BIPAP principle. ASV is indicated in the presence of CSR on PSG in patients with predominant congestive HF (EF <40\%) (2). However, it has been reported that ASV treatment is indicated for normalizing $\mathrm{AHI}$ and for the treatment of CSA syndrome associated with CHF (23). In a non-randomized study, 11 patients were treated with ASV in addition to medical treatment for 6 months, and only medical treatment was applied to the comparison group. At the end of 6 months, AHI hourly event decreased significantly compared to the baseline $(p=0.001)$ and there was also a significant improvement in sleep efficiency $(p=0.01), \operatorname{LVEF}(p=0.04)$, daytime sleepiness ( $p=0.001)$ and quality of life $(p=0.005)$ in the ASV group (28).

BIPAP is not the first treatment option in patients with OSA, but it is stated that it can be the first choice in non-invasive methods in patients with hypoxemia-hypoventilation syndromes who can not tolerate CPAP treatment and who are not able to exhale against high pressure (2). As a result of the evaluations, only one study has applied BIPAP therapy in CHF patients with OSA and improvement was observed in LVEF, quality of life and functional capacity (8). 
American Academy of Sleep Medicine (2011) states that administered oxygen therapy at night is indicated for the treatment of CSA in patients with CHF (29). In patients with HF, additional nocturnal oxygen therapy is administered through nasal cannula for the treatment of CSA. Data from some small-sample, short-term studies suggest that oxygen therapy with nasal cannula improves AHI, exercise capacity and LVEF, and decreases BNP level and sympathetic nervous system activity (23). In a small sample study with CHF patients with CSA (LVEF $<45 \%$, mean age 65 years, $\mathrm{AHI}>5$ /hour), oxygen therapy at night was shown to improve exercise capacity, cardiac function, and cardiac sympathetic nerve activity (30). In the light of the findings of our study, two studies in the literature $(6,19)$ reported that oxygen therapy for the treatment of CHF patients with CSA improved SRD (6,19), LVEF (19) and had a positive effect on ventricular arrhythmias (19). One of the studies showed that oxygen therapy improved quality of life and functional capacity (17), but the other study (6) did not achieve the same effect (Table 2). When the literature is examined, it has been shown that night oxygen therapy through nasal cannula have no positive effect on daytime sleepiness, sleep quality, quality of life or cognitive functions. Unlike CPAP and ASV, nighttime oxygen therapy was found not effective in eliminating upper airway obstruction, which may be accompanied by central apneas. In the light of these findings, it is stated that oxygen therapy can be used only if pressure-assisted treatments are ineffective or patients cannot tolerate these treatments (23,31-33).

In the literature, it has been hypothesized that atrial overdrive pacemaker therapy can increase cardiac filling and decrease pulmonary obstruction by increasing the heart rate, thus reduce or prevent CSA formation. In some small sample studies, atrial overdrive pacemaker therapy has been shown to reduce the number of CSA attacks, improve oxygen saturation, and reduce stimulation in HF patients $(23,34,35)$. In another study, it was reported that atrial overdrive pacemaker treatment in CSA did not provide any improvement (36). In this study, it was determined that atrial overdrive pacemaker treatment in CHF patients with OSA provided significant reduction in AHI in a single RCT (Table 2) (21).

\section{Conclusion}

Sleep disorder is a common problem in HF patients. In the studies performed within the systematic review, the effectiveness of medical treatments and interventions for sleep disturbance in $\mathrm{HF}$ patients were evaluated. When the efficacy of the interventions was examined, it was observed that the addition of CPAP to medical therapy improved CSA and nocturnal oxygenation and increased 6MWD. The use of CPAP improved daytime sleepiness and LVEF with regular use in HF patients with OSA, but no effect on quality of life was observed. ASV treatment added to medical therapy also improved CSA and OSA with regular use. ASV alone was effective in reducing respiratory distress and sleep apnea, and it reduced $A H I$, improved LVEF, and reduced ventricular pulse. The use of ASV increased 6MWD compared to CPAP. When CPAP and BIPAP were evaluated, CPAP was effective in improving sleep quality and quality of life, while BIPAP was more effective in improving quality of life, functional capacity and BP. In addition to these interventions, the physical exercise program has been shown to be effective in improving sleep quality.

\section{Ethics}

Peer-review: External and internal peer-reviewed.

Author Contributions: Concept - H.U., H.B.O.; Design - H.U., H.B.O.; Supervision - H.U.; Resources - H.U., H.B.O.; Materials - H.U., H.B.O.; Data Collection and/or Processing - H.U., H.B.O.; Analysis and/or Interpretation - H.U., H.B.O.; Literature Search - H.U., H.B.O.; Writing Manuscript - H.U., H.B.O.; Critical Review - H.U.

Conflict of Interest: No conflict of interest was declared by the authors.

Financial Disclosure: The authors declared that this study received no financial support.

\section{References}

1. McMurray JJV, Adamopoulos S, Anker SD, Auricchio A, Bohm M, Dickstein K, et al. Akut ve kronik kalp yetersizliği tanı ve tedavisine yönelik 2012 ESC kılavuzu. Türk Kardiyol Dern Arș 2012;(Özel Sayı 3): 1-128.

2. Fırat H. OSAS’ta PAP tedavisi ve cihaz seçimi. Güncel göğüs hastalıkları derisi 2014; 2: 184-91.

3. Demir A, Ursavaş A, Tana Aslan A, Gülbay B, Çiftçi B, Çuhadaroğlu Ç, et al. Türk Toraks Derneği Obstrüktif Uyku Apne Sendromu Tanı ve Tedavi Uzlașı Raporu. Türk Toraks Dergisi 2012; 13(Suppl 1): 1-65.

4. Karaçam Z. Sistematik derleme metodolojisi: Sistematik derleme hazırlamak için bir rehber. DEUHYO ED 2013; 6: 26-33.

5. The joanna briggs institute critical appraisal tools for use in JBI. Checklist for randomized controlled Trials. Joanna Briggs Institute 2017: 1-9.

6. Staniforth AD, Kinnear WJ, Starling R, Hetmanski DJ, Cowley AJ. Effect of oxygen on sleep quality, cognitive function and sympathetic activity in patients with chronic heart failure and Cheyne-Stokes respiration. Eur Heart J 1998; 19: 922-8.

7. Egea CJ, Aizpuru F, Pinto JA, Ayuela JM, Ballester E, Zamarrón C, et al. Cardiac function after CPAP therapy in patients with chronic heart failure and sleep apnea: A multicenter study. Sleep Med 2008; 9: 660-6.

8. Khayat RN, Abraham WT, Patt B, Roy M, Hua K, Jarjoura D. Cardiac effects of continuous and bilevel positive airway pressure for patients with heart failure and obstructive sleep apnea: A Pilot Study. Chest 2008; 134: 1162-8.

9. Bradley TD, Logan AG, Kimoff RJ, Sériès F, Morrison D, Ferguson K, et al. Continuous positive airway pressure for central sleep apnea and heart failure. N Engl J Med 2005; 353: 2025-33.

10. O'Connor CM, Whellan DJ, Fiuzat M, Punjobi NM, Tasissa G, Anstrom KJ, et al. Cardiovascular outcomes with minute ventilation-targeted adaptive servoventilation therapy in heart failure. The CAT-HF Trial. J Am Coll Cardiol 2017; 69: $1577-87$.

11. Arzt M, Schroll S, Series F, Lewis K, Benjamin A, Escourrou P, et al. Autoservoventilation in heart failure with sleep apnoea: a randomised controlled trial. Eur Respir J 2013; 42: 1244-54.

12. Randerath WJ, Nothofer G, Priegnitz C, Anduleit N, Treml M, Kehl V, et al. Long-term auto-servoventilation or constant positive pressure in heart failure and coexisting central with obstructive sleep apnea. CHEST 2012; 142: 440-7.

13. Hetzenecker A, Escourrou P, Kuna ST, Series F, Lewis K, Birner C, et al. Treatment of sleep apnea in chronic heart failure patients with auto-servo ventilation improves sleep fragmentation: a randomized controlled trial. Sleep Med 2016; 17: 25-31.

14. Cowie MR, Woehrle H, Wegscheider K, Angermann C, d'Ortho MP, Erdmann E, et al. Adaptive servo-ventilation for central sleep apnea in systolic heart failure. N Engl J Med 2015; 373: 1095-105.

15. Kasai T, Kasagi S, Maeno K, Dohi T, Kawana F, Kato M, et al. Adaptive servoventilation in cardiac function and neurohormonal status in patients with 
heart failure and central sleep apnea non-responsive to continuous positive airway pressure. JACC Heart Fail 2013; 1: 58-63.

16. Priefert HJ, Hetzenecker A, Escourrou P, Luigart R, Series F, Lewis K, et al. Effects of adaptive servo-ventilation on ventricular arrhytmias in patients with stable congestive heart failure and sleep-disordered breathing. Subanalysis of a randomized controlled trail. Somnologie 2017; 21: 19-27.

17. Kawecka-Jaszcz K, Bilo G, Drozdz T, Debicka-Dabrowska D, Kiełbasa G, Malfatto $\mathrm{G}$, et al. Effects of device guided slow breathing training on exercise capacity, cardiac function, and respiratory patterns during sleep in male and female patients with chronic heart failure. Pol Arch Intern Med 2017; 127: 8-15.

18. Smith LA, Vennelle M, Gardner RS, McDonagh TA, Denvir MA, Douglas NJ, et al. Auto-titrating continuous positive airway pressure therapy in patients with chronic heart failure and obstructive sleep apnoea: a randomized placebocontrolled trial. Eur Heart J 2007; 28: 1221-7.

19. Nakao YM, Ueshima K, Yasuno S, Sasayama S. Effects of nocturnal oxygen therapy in patients with chronic heart failure and central sleep apnea: CHFHOT study. Heart Vessels 2016; 31: 165-72.

20. Suna JM, Mudge A, Stewart I, Marquart L, O’Rourke P, Scott A. The effect of a supervised exercise training programme on aleep quality in recently discharged heart failure patients. Eur J Cardiovasc Nurs 2015; 14: 198-205.

21. Sharafkhaneh A, Sharafkhaneh H, Bredikus A, Guilleminault C, Bozkurt B, Hirshkowitz M. Effect of atrial overdrive pacing on obstructive sleep apnea in patients with systolic heart failure. Sleep Med 2007; 8: 31-6.

22. Lyons OD, Floras JS, Logan AG, Beanlands R, Cantolla JD, Fitzpatrick M, et al. Design of the effect of adaptive servo-ventilation on survival and cardiovascular hospital admissions in patients with heart failure and sleep apnoea: the ADVENT-HF trial. Eur J Heart Fail 2017; 19: 579-87.

23. Costanzo MR, Khayat R, Ponikowski P, Augostini R, Stellbrink C, Mianulli M, et al. Mechanisms and clinical consequences of untreated central sleep apnea in heart failure. J Am Coll Cardiol 2015; 65: 72-84

24. Yancy CW, Jessup M, Bozkurt B, Butler J, Casey DE Jr, Drazner MH, et al. 2013 ACCF/AHA guideline for the management of heart failure: a report of the American College of Cardiology Foundation/American Heart Associaion Task Force on Practice Guidelines. J Am Coll Cardiol 2013; 62: 147-239.

25. Heart Failure Society of America, Lindenfeld J, Albert NM, Boehmer JP, Collins SP, Ezekowitz JA, et al. HFSA 2010 comprehensive heart failure practice guideline. J Card Fail 2010; 16: 1-194.
26. Mohsenin V. Obstructive sleep apnea and hypertension: A Critical Review. Curr Hypertens Rep 2014; 16: 482-7.

27. Hastings PC, Vazir A, Meadows GE, Dayer M, Poole-Wilson PA, Mclntyre HF, et al. Adaptive servo-ventilation in heart failure patients with sleep-apnea: A real world study. Int J Cardiol 2010; 139: 17-24.

28. Philippe C, Stoica-Hernan M, Drouot X, Raffestin B, Escourrou P, Hittinger L, et al. Compliance with and effectiveness of adaptiveservo ventilation versus continuous positive respiration in heart failure over a six month period. Heart 2006; 92: 337-42.

29. Aurora RN, Chowdhuri S, Ramar K, Bista SR, Casey KR, Lamm Cl, et al. The treatment of central sleep apnea syndromes in adults: practice parameters with an evidencebased literature review and meta-analyses. Sleep 2012; 35: $17-40$.

30. Toyama T, Seki R, Kasama S, Isobe N, Sakurai S, Adachi H, et al. Effectiveness of nocturnal home oxygen therapy to improve exercise capacity, cardiac function and cardiac sympatetic nerve activity in patients with chronic heart failure and central sleep apnea. Circ J 2009; 73: 299-304.

31. Krachman SL, D’Alonso GE, Berger TJ, Eisen HJ. Comparison of oxygen therapy with nasal continuous positive airway pressure on Cheyne-Stokes respiration during sleep in congestive heart failure. Chest 1999; 116: 1550-7.

32. Campbell AJ, Ferrier K, Neill AM. Effect of oxygen versus adaptive pressure support servo-ventilation in patients with central sleep apnoea-CheyneStokes respiration and congestive heart failure. Intern Med J 2012; 42: 1130-6.

33. Arzt M, Floras JS, Logan AG, Kimoff RJ, Series F, Morrison D, et al. Suppression of central sleep apnea by continuous positive airway pressure and transplantfree survival in heart failure: a post hoc analysis of the Canadian Positive Airway Pressure for Patients with Central Sleep Apnea and Heart Failure Trial (CANPAP). Circulation 2007; 115: 3173-80.

34. Garrigue S, Bordier P, Jais P, Shah DC, Hocini M, Raherison C, et al. Benefit of atrial pacing in sleep apnea syndrome. N Engl J Med 2002; 346: 404-12.

35. Weng CL, Chen Q, Ma YL, He QY. A meta analysis of the effects of atrial overdrive pacing on sleep apnea syndrome. Pacing Clin Electrophysiol 2009; 32: $1434-43$

36. Luthje L, Unterberg-Buchwald C, Dajani D, Vollmann D, Hassesfuss G, Andreas S. Atrial overdrive pacing in patients with sleep apnea with implanted pacemaker. Am J Respir Crit Care Med 2005; 172: 118-22. 